\title{
THE RADIAL VELOCITY TATOOINE SEARCH FOR CIRCUMBINARY PLANETS: PLANET DETECTION LIMITS FOR A SAMPLE OF DOUBLE-LINED BINARY STARS—INITIAL RESULTS FROM KECK I/HIRES, SHANE/CAT/HAMSPEC, AND TNG/SARG OBSERVATIONS
}

\author{
Maciej Konacki ${ }^{1,2}$, Matthew W. Muterspaugh ${ }^{3,4}$, Shrinivas R. Kulkarni ${ }^{5}$, and Krzysztof G. Heeminiak ${ }^{1}$ \\ ${ }^{1}$ Nicolaus Copernicus Astronomical Center, Polish Academy of Sciences, Rabianska 8, 87-100 Torun, Poland; maciej@ncac.torun.pl \\ ${ }^{2}$ Astronomical Observatory, A. Mickiewicz University, Sloneczna 36, 60-286 Poznan, Poland \\ ${ }^{3}$ Department of Mathematics and Physics, College of Arts and Sciences, Tennessee State University, Boswell Science Hall, Nashville, TN 37209 , USA \\ ${ }^{4}$ Tennessee State University, Center of Excellence in Information Systems, 3500 John A. Merritt Blvd., Box No. 9501, Nashville, TN 37203-3401, USA \\ ${ }^{5}$ Division of Physics, Mathematics and Astronomy, California Institute of Technology, Pasadena, CA 91125, USA \\ Received 2009 June 18; accepted 2009 August 27; published 2009 September 23
}

\begin{abstract}
We present preliminary results of the first and on-going radial velocity survey for circumbinary planets. With a novel radial velocity technique employing an iodine absorption cell, we achieve an unprecedented radial velocity (RV) precision of up to $2 \mathrm{~m} \mathrm{~s}^{-1}$ for double-lined binary stars. The high-resolution spectra collected with the Keck I/Hires, TNG/Sarg, and Shane/CAT/Hamspec telescopes/spectrographs over the years 2003-2008 allow us to derive RVs and compute planet detection limits for 10 double-lined binary stars. For this initial sample of targets, we can rule out planets on dynamically stable orbits with masses as small as $\sim 0.3$ to $3 M_{\text {Jup }}$ for the orbital periods of up to $\sim 5.3$ years. Even though the presented sample of stars is too small to make any strong conclusions, it is clear that the search for circumbinary planets is now technique-wise possible and eventually will provide new constraints for the planet formation theories.
\end{abstract}

Key words: binaries: spectroscopic - planetary systems - stars: individual (HD9939, HD13974, HD47415, HD78418, HD86146, HD195987, HD210027, HD214686, HD221950, HD282975) - techniques: radial velocities

Online-only material: color figures

\section{INTRODUCTION}

Searches for planets in close binary systems explore the degree to which stellar multiplicity inhibits or promotes planet formation (Muterspaugh 2005; Muterspaugh et al. 2006, 2007). Detection of giant planets orbiting both components of short period ( $P<60$ days) binaries (circumbinary planets) will have significant consequences for theoretical understandings of how giant planets are formed. The binarity of the central body creates an environment in which the evolution of a protoplanetary disk is substantially different than around single stars (Artymowicz \& Lubow 1994). This must have an effect on the migration of giant planets in a disk as well as on the "parking" mechanism and their final orbit. Likely, also the dynamical interaction between protoplanets and the planets in a multi-planet system should be affected by the central body binarity and presumably result in a different distribution of the orbital elements of planets. Finally, if one assumes that planetary orbits are coplanar with the orbit of an eclipsing binary, then there is an enhanced probability of detecting a circumbinary transiting planet (Konacki 2009; Ofir 2009, 2008; Schneider 1994; Schneider \& Chevreton 1990).

The recent work by Konacki (2005a, 2009) demonstrates a method for obtaining radial velocity (RV) precisions of up to $5 \mathrm{~m} \mathrm{~s}^{-1}$ for double-lined spectroscopic binaries (SB2s; now improved to $2 \mathrm{~m} \mathrm{~s}^{-1}$ ), and for the first time opens the opportunity to search for circumbinary planets via RVs. This method has been applied to a new search for circumbinary planets: The Attempt To Observe Outer-planets In Non-singlestellar Environments (TATOOINE).

Planets in binary and multiple stars have been a subject of theoretical works long before any extrasolar planets were detected. Dvorak (1984) investigated dynamical stability of a wide range of planetary configurations in the framework of the elliptic restricted three-body problem. These included the so-called P-type (planet-type; circumbinary orbits), S-type (satellite-type; circumprimary or circumsecondary orbits) and L-type orbits (Librator-type; orbits around stable Lagrangian points L4 or L5 for the mass ratios $\mu<0.04)$. This subject was continued by, e.g., Holman \& Wiegert (1999) who based on extensive numerical simulations provided useful formulas allowing one to compute stable regions for among others circumbinary planets.

Circumbinary disks and planet formation in such disks have been of some interest to theorists as well. The evolution of a circumbinary disk is studied by Artymowicz \& Lubow (1994) who demonstrates that such a disk will be truncated at its inner edge by tidal torques to within 1.8-2.6 times the semimajor axis of the binary. Moriwaki \& Nakagawa (2004) and Scholl et al. (2007) show that planetesimal accretion should be possible in circumbinary disks, and Quintana \& Lissauer (2006) provide numerical proofs that planetary systems similar to those around single stars may be formed around binary stars with the apastron distance $\leqslant 0.2 \mathrm{AU}$. Most recently, in a series of papers, Pierens \& Nelson (2007, 2008a, 2008b) investigated formation, migration, and evolution of planets in circumbinary disks. In particular, they suggest that circumbinary planets may be more common in the Saturn-mass regime as higher mass planets are more likely to undergo close encounters with the secondary star (Pierens \& Nelson 2008b).

There are a few known cases of young spectroscopic binaries with circumbinary disks. These include AK Sco (Andersen et al. 1989), GW Ori (Mathieu et al. 1991), DQ Tau (Mathieu et al. 1997), and GG Tau (Dutrey et al. 1994). The observations confirm the truncation of the disks at their inner edge as predicted by theory. Recently, Kastner et al. (2008) have detected $\mathrm{CO}, \mathrm{HCN}, \mathrm{CN}$, and $\mathrm{HCO}^{+}$around a $\sim 12 \mathrm{Myr}$ old 
close binary V4046 Sgr demonstrating that it is surrounded by a rich molecular disk and showing a great similarity to the $\sim 8 \mathrm{Myr}$ old star TW Hya; a star/disk system regarded a representative of the early solar nebula. Finally, Zuckerman et al. (2008) provide evidence that an SB2 BD+20 307 is an old binary star surrounded by a debris disk likely formed in a collision involving a terrestrial planet.

Clearly, there is enough evidence that circumbinary planets should form, evolve, and survive on stable orbits around close binary stars. Yet no radial velocity survey has been carried out to detect such planets despite the fact that the RV technique for single stars has allowed for a thriving scientific endeavor over the last 15 years. The fundamental problem with double-lined spectroscopic binary stars is that their spectra are highly variable due to the orbital motion of their components resulting in Doppler shifts typically up to $\sim 100 \mathrm{~km} \mathrm{~s}^{-1}$ for each component spectrum. Hence, the approach used for single stars where a Doppler shift of an otherwise constant shape (spectrum) is found cannot be applied.

In this paper, we present the preliminary results of the first radial velocity survey for circumbinary planets. In Section 2 , we discuss the novel iodine cell based approach that allows us to precisely measure RVs of SB2s. In Section 3, we describe in more detail our on-going RV effort to detect circumbinary planets. In Section 4, we show the planet detection limits for 10 SB2s from our survey; and conclude in Section 5.

\section{PRECISION RVs OF DOUBLE-LINED SPECTROSCOPIC BINARIES}

In the iodine cell $\left(I_{2}\right)$ technique, the Doppler shift of a star spectrum $\Delta \lambda$ is determined by solving the following equation (Marcy \& Butler 1992):

$$
I_{\mathrm{obs}}(\lambda)=\left[I_{s}\left(\lambda+\Delta \lambda_{s}\right) T_{I_{2}}\left(\lambda+\Delta \lambda_{I_{2}}\right)\right] \otimes \mathrm{PSF},
$$

where $\Delta \lambda_{s}$ is the shift of the star spectrum, $\Delta \lambda_{I_{2}}$ is the shift of the iodine transmission function $T_{I_{2}}, \otimes$ represents a convolution, and PSF is a spectrograph's point-spread function. The parameters $\Delta \lambda_{s}$ and $\Delta \lambda_{I_{2}}$, as well as parameters describing the PSF, are determined by performing a least-squares fit to the observed (through the iodine cell) spectrum $I_{\mathrm{obs}}$. For this purpose, one also needs (1) a high signal-to-noise ratio $(\mathrm{S} / \mathrm{N})$ star spectrum taken without the cell $I_{s}$, which serves as a template for all the spectra observed through the cell and (2) the $I_{2}$ transmission function $T_{I_{2}}$ obtained, for example, with the Fourier Transform Spectrometer at the Kitt Peak National Observatory. The Doppler shift of a star spectrum is then given by $\Delta \lambda=\Delta \lambda_{s}-\Delta \lambda_{I_{2}}$. Such an iodine technique can only be applied to single stars. This is dictated by the need to supply an observed template spectrum of each component of a target binary star in Equation (1).

In the case when a composite spectrum of a binary star is observed, the classical approach with the iodine cell cannot be used since it is not possible to observationally obtain two separate template spectra of the binary components. This problem can be resolved as follows. First, two sequential exposures of each (binary) target are always taken - one with and the other without the cell. This way one obtains an instantaneous template that is used to model only the adjacent exposure taken with the cell. The derived Doppler shift, $\Delta \lambda_{i}$ (where $i$ denotes the epoch of the observation), carries no meaning, since each time a different template is used. Moreover, it describes a Doppler "shift" of a composed spectrum that is typically different at each epoch.
However, the parameters - in particular, the wavelength solution and the parameters describing PSF — are accurately determined and can be used to extract the star spectrum, $I_{\mathrm{obs}}^{\star, i}(\lambda)$, for each epoch $i$ :

$$
I_{\mathrm{obs}}^{\star, i}(\lambda)=\left[I_{\mathrm{obs}}^{i}(\lambda) \otimes^{-1} \mathrm{PSF}^{i}\right] / T_{I_{2}}(\lambda),
$$

where $\otimes^{-1}$ denotes deconvolution, and $\mathrm{PSF}^{i}$ represents the set of parameters describing PSF at the epoch $i$. Such a star spectrum has an accurate wavelength solution, is free of the $I_{2}$ lines and the influence of a varying PSF.

In the next step, the velocities of both components of a binary target are measured with the well-known two-dimensional cross-correlation technique TODCOR (Zucker \& Mazeh 1994), using as templates the synthetic spectra derived with the ATLAS 9 and ATLAS 12 programs (Kurucz 1995) and matched to the observed spectrum, $I_{s}(\lambda)$. This approach results in an RV precision of 20-30 $\mathrm{m} \mathrm{s}^{-1}$ (Konacki 2005a). Now, having the RVs obtained with TODCOR, one can carry out a tomographic disentangling of the composite spectra of a binary to produce the real (observed) component spectra and use them in the original Equation (1). This finally allows us to directly measure the RVs without relying on synthetic templates. Such a procedure results in RVs having a precision of up to several $\mathrm{m} \mathrm{s}^{-1}$ (Konacki 2009). The formal errors of the velocities are derived from the scatter between the velocities from different echelle orders.

\section{THE TATOOINE SURVEY}

The TATOOINE survey was initiated in mid 2003 with the $10 \mathrm{~m}$ Keck I and its Hires spectrograph (Vogt et al. 1994) as an addition to an RV survey for planets orbiting components of speckle binary and multiple stars (Konacki 2005b). The original sample of $12 \mathrm{SB} 2 \mathrm{~s}$ was primarily selected to provide precision RVs for the astrometric data collected at the Palomar Testbed Interferometer (Colavita et al. 1999; Konacki \& Lane 2004) and to test the new RV technique on a sample of close binaries. The Keck I/Hires survey was continued until mid 2007. The sample was also monitored from mid 2006 until mid 2007 with the $3.6 \mathrm{~m}$ Telescopio Nazionale Galileo (TNG) and its Sarg spectrograph (Gratton et al. 2001). Since fall 2006, the TATOOINE project has been collecting velocity measurements at Lick Observatory using the $3 \mathrm{~m}$ Shane and $0.6 \mathrm{~m}$ Coude Auxiliary Telescopes (CATs) and the Hamilton Spectrograph (Hamspec; Vogt 1987). Overall, 50 northern binaries are currently being monitored by the TATOOINE project. Recently, we have also initiated a southern effort at the $3.9 \mathrm{~m}$ Anglo-Australian Telescope (AAT) with the UCLES spectrograph (Diego et al. 1990).

The spectrographs employed in the survey, Hires, Sarg, and Hamspec, are all high-resolution echelle spectrographs equipped with iodine absorption cells. They provide spectra with a resolution of $67,000,86,000$, and 60,000 , respectively, across a $\sim 400-900 \mathrm{~nm}$ bandwidth centered on the 500-600 nm range corresponding to an iodine cell's spectral features. The cells are used to superimpose a reference absorption spectrum in a manner described in Section 2. For each measurement, back-to-back spectra of the target binary were acquired, first with an iodine absorption cell in the path of the starlight, then with the iodine cell removed. Depending on the telescope, different exposure times were used to balance the resulting $\mathrm{S} / \mathrm{N}$ per collapsed pixel and the efficient use of telescope's time. The highest $\mathrm{S} / \mathrm{N}$ of $\sim 250$ was achieved with the Keck $\mathrm{I} /$ Hires also to test the data pipeline in the regime of high $\mathrm{S} / \mathrm{N}$ spectra. The typical $\mathrm{S} / \mathrm{N}$ was $\sim 75-150$ for the $\mathrm{TNG} / \mathrm{Sarg}$ and 

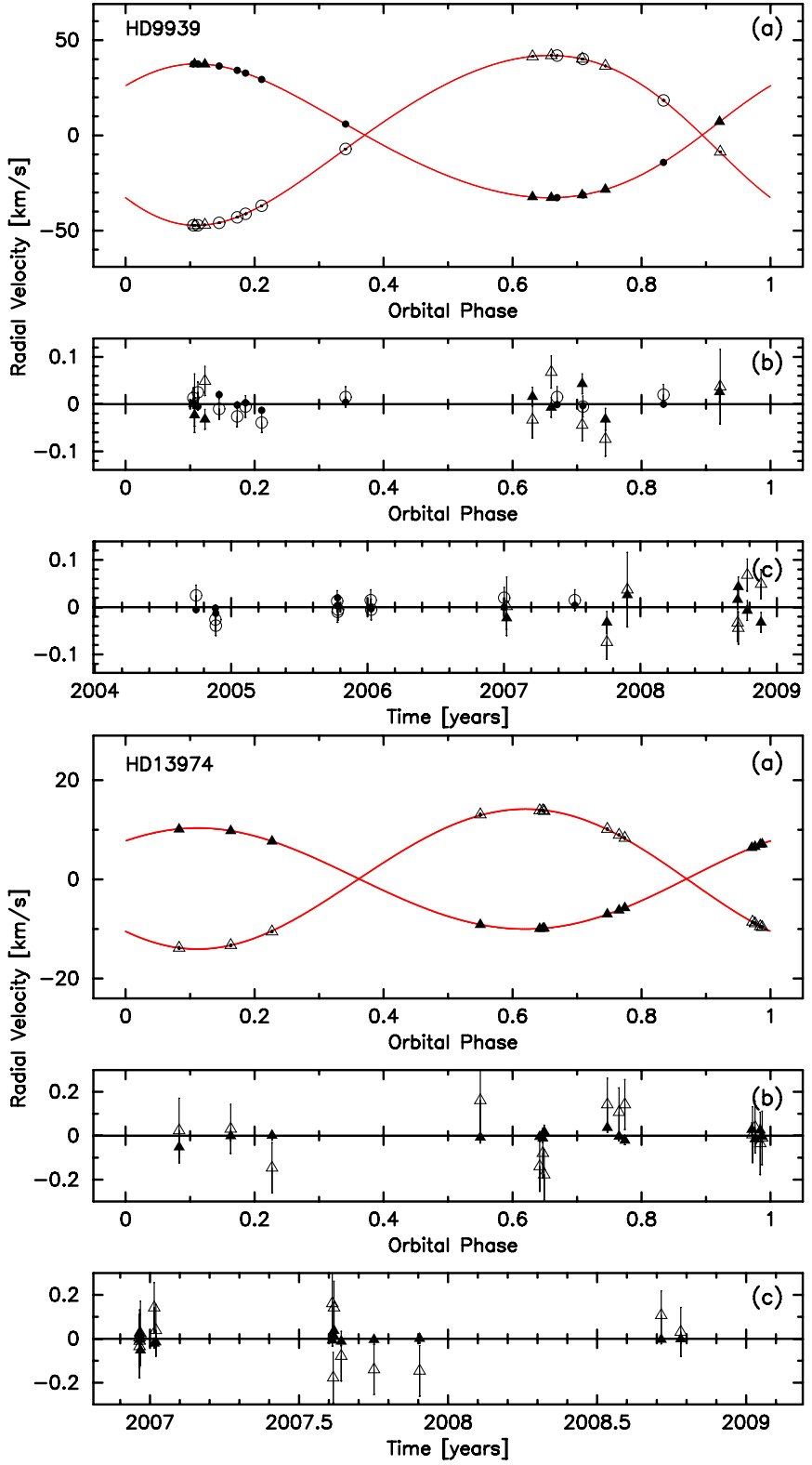

Figure 1. RVs of HD9939 (top) and HD13974 (bottom) as a function of the orbital phase (a), and the residuals (observed minus modeled RVs) as a function of the orbital phase (b) and time (c). The primary is denoted with filled symbols, the secondary with open ones, and the best-fit RV model with a solid line. The Keck I/Hires is denoted with circles, Shane/CAT/Hamspec with triangles, and TNG/Sarg with stars.

(A color version of this figure is available in the online journal.)

50-150 for the Shane/CAT/Hamspec. In consequence, the RVs have different precision ranging from $~ 2$ (HD195987, the best case) to $20 \mathrm{~m} \mathrm{~s}^{-1}$ for the primary stars. In any case, the precision is sufficient to detect planets with masses as small as $0.3 M_{\text {Jup }}$. Note also that due to a brightness ratio between the primary and secondary, the RVs of the secondary are typically of lower precision as the $\mathrm{S} / \mathrm{Ns}$ are for the composite observed spectra. For example, an $\mathrm{S} / \mathrm{N}$ of 250 and a brightness ratio of 6.7 (HD195987) corresponds to an S/N of 220 for the primary and only 30 for the secondary.

A circumbinary planet will exhibit two indirect effects on the RV of the stars. First, the apparent system velocity will vary in a periodic manner due to the motion of the binary about the
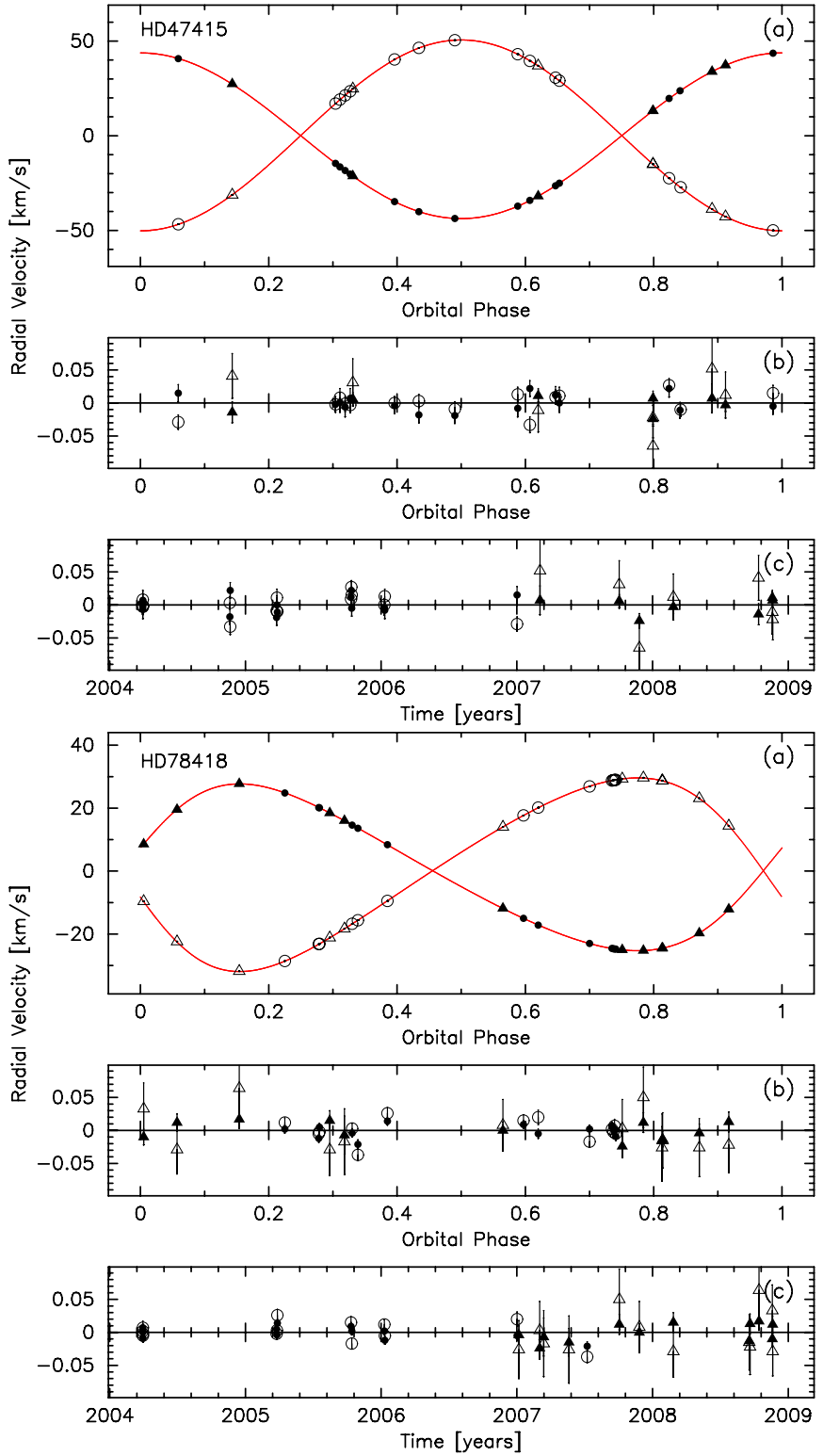

Figure 2. RVs of HD47415 (top) and HD78418 (bottom) as a function of the orbital phase (a), and the residuals (observed minus modeled RVs) as a function of the orbital phase (b) and time (c). The primary is denoted with filled symbols, the secondary with open ones, and the best-fit RV model with a solid line. The Keck I/Hires is denoted with circles, Shane/CAT/Hamspec with triangles, and TNG/Sarg with stars.

(A color version of this figure is available in the online journal.)

system barycenter, with amplitude (Muterspaugh et al. 2007)

$$
\Delta v_{b}=57 \mathrm{~m} \mathrm{~s}^{-1} \times \frac{\left(M_{p} / M_{\mathrm{Jup}}\right) \sin i_{p}}{\sqrt{\left(\left(M_{b}+M_{p}\right) / M_{\odot}\right)\left(a_{p} / 1 \mathrm{AU}\right)}} .
$$

Differential reflex motion and perturbations of the binary orbit by the planetary companion are expected to be negligible on reasonable timescales. Second, the finite speed of light will cause apparent changes in the phase of the binary orbit due to the reflex motion of the binary about the system center of mass. This phase shift is detected for planets with masses as small as (Muterspaugh et al. 2007)

$$
M_{p}=70 M_{\text {Jup }} \times \frac{\left(\sigma_{r v} / 20 \mathrm{~m} \mathrm{~s}^{-1}\right)\left(P_{b} / 5 \mathrm{~d}\right)^{4 / 3}\left(M_{b} / M_{\odot}\right)^{2 / 3}}{\sqrt{N-6} \sin i_{b} \sin i_{p}\left(a_{p} / 1 \mathrm{AU}\right)},
$$



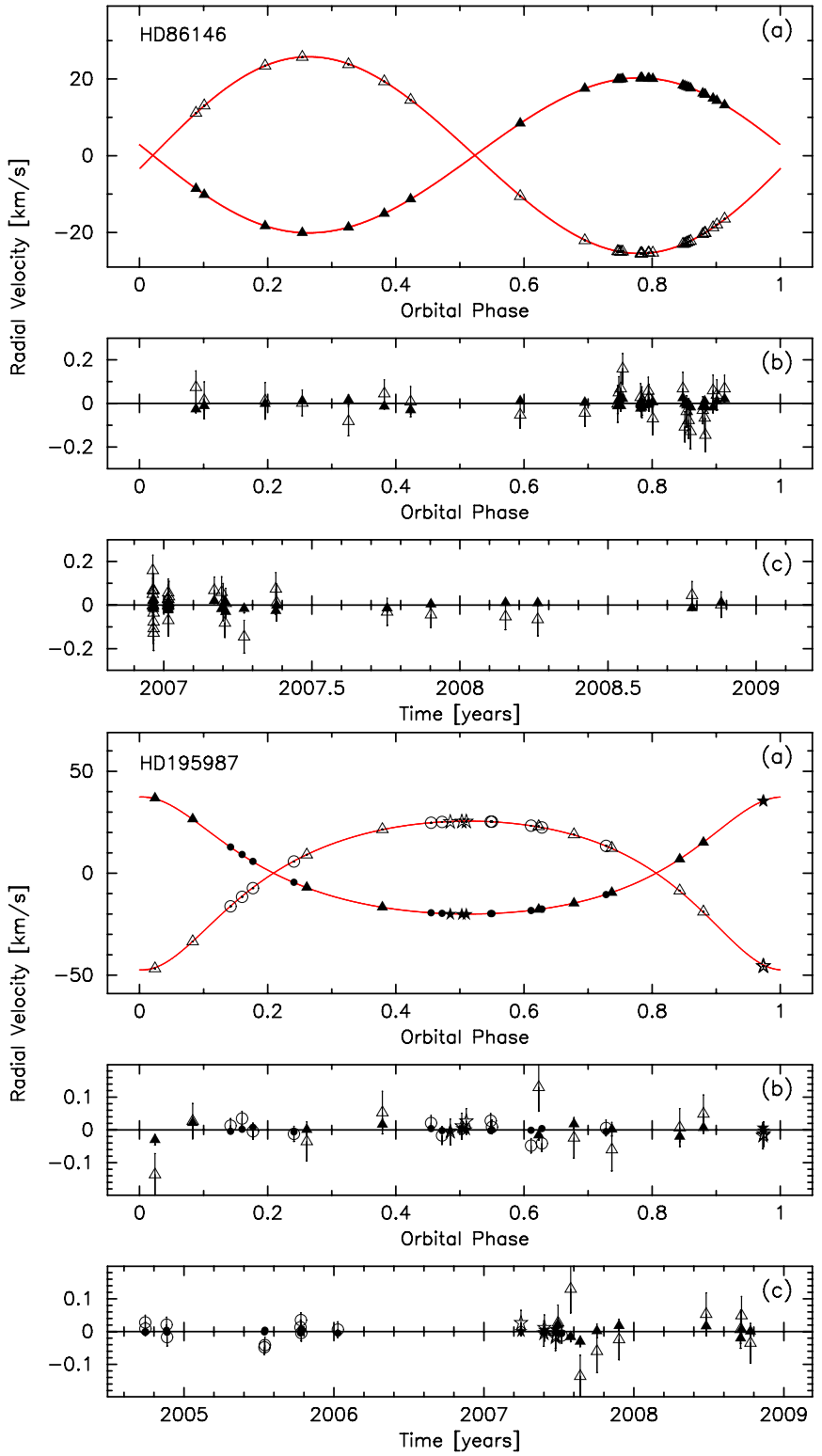

Figure 3. RVs of HD86146 (top) and HD195987 (bottom) as a function of the orbital phase (a), and the residuals (observed minus modeled RVs) as a function of the orbital phase (b) and time (c). The primary is denoted with filled symbols, the secondary with open ones, and the best-fit RV model with a solid line. The Keck I/Hires is denoted with circles, Shane/CAT/Hamspec with triangles, and TNG/Sarg with stars.

(A color version of this figure is available in the online journal.)

assuming $1 \%$ false alarm probability (FAP; $\mathrm{S} / \mathrm{N}=5.8$ ) where $N$ is the number of observations, and $i_{b}$ and $i_{p}$ are the inclinations of the binary and planet orbits, respectively. Note that this observable is actually more sensitive to longer period planets. In combination, these effects allow us to detect Jupiter mass planets or smaller for an extended range of orbital periods. In the current analysis, the second effect has been ignored as it is small on the relatively short timescales being considered.

\section{PLANET DETECTION LIMITS FOR 10 SB2s}

For this initial sample of stars, we have selected these SB2s from our survey that have long time span RV data sets and an orbital phase coverage allowing for a reliable tomographic disentangling. These are HD 9939, HD 13974 (Figure 1),
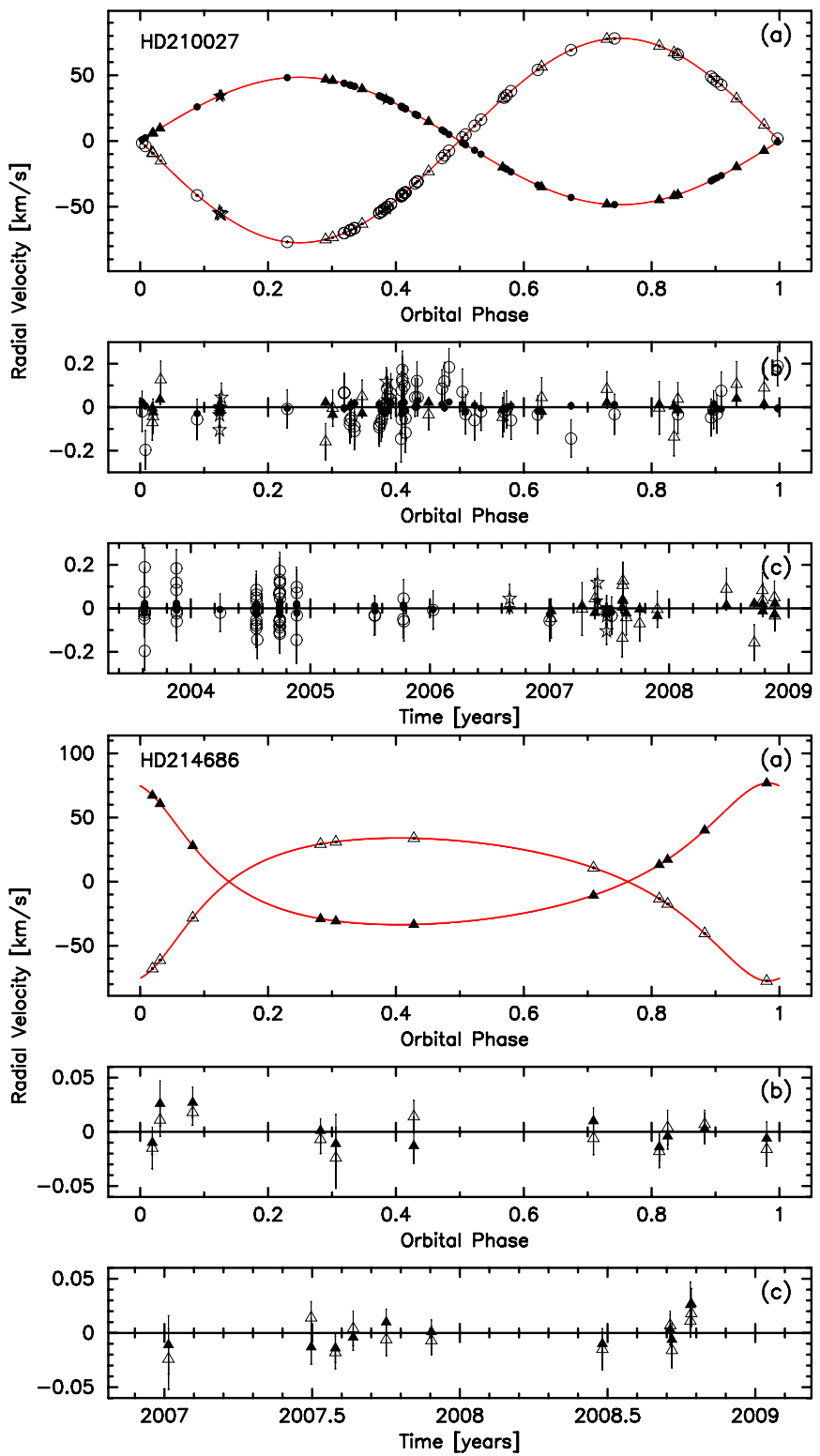

Figure 4. RVs of HD210027 (top) and HD214686 (bottom) as a function of the orbital phase (a), and the residuals (observed minus modeled RVs) as a function of the orbital phase (b) and time (c). The primary is denoted with filled symbols, the secondary with open ones, and the best-fit RV model with a solid line. The Keck I/Hires is denoted with circles, Shane/CAT/Hamspec with triangles, and TNG/Sarg with stars.

(A color version of this figure is available in the online journal.)

HD 47415, HD 78418 (Figure 2), HD 86146, HD 195987 (Figure 3), HD 210027, HD 214686 (Figure 4), HD 221950, and HD 282975 (Figure 5). Let us note that we recently made improvements to our data pipeline, and the Keck I/Hires RVs used in this paper are typically several $\mathrm{m} \mathrm{s}^{-1}$ more accurate than those shown in Konacki (2009). The internal RV errors are computed from the scatter between the echelle orders used in the reduction. They are expected to underestimate the real RV scatter. In addition to the stellar jitter, one of the main reasons for the underestimation of the errors are the imperfections in the disentangled template spectra. While these imperfections are impossible to see with a "naked eye," they still contribute to the total error budget. For this reason, we add an additional error in quadrature to obtain a reduced $\chi^{2}$ equal to 1 for a simple Keplerian model. The RVs are first modeled with a 

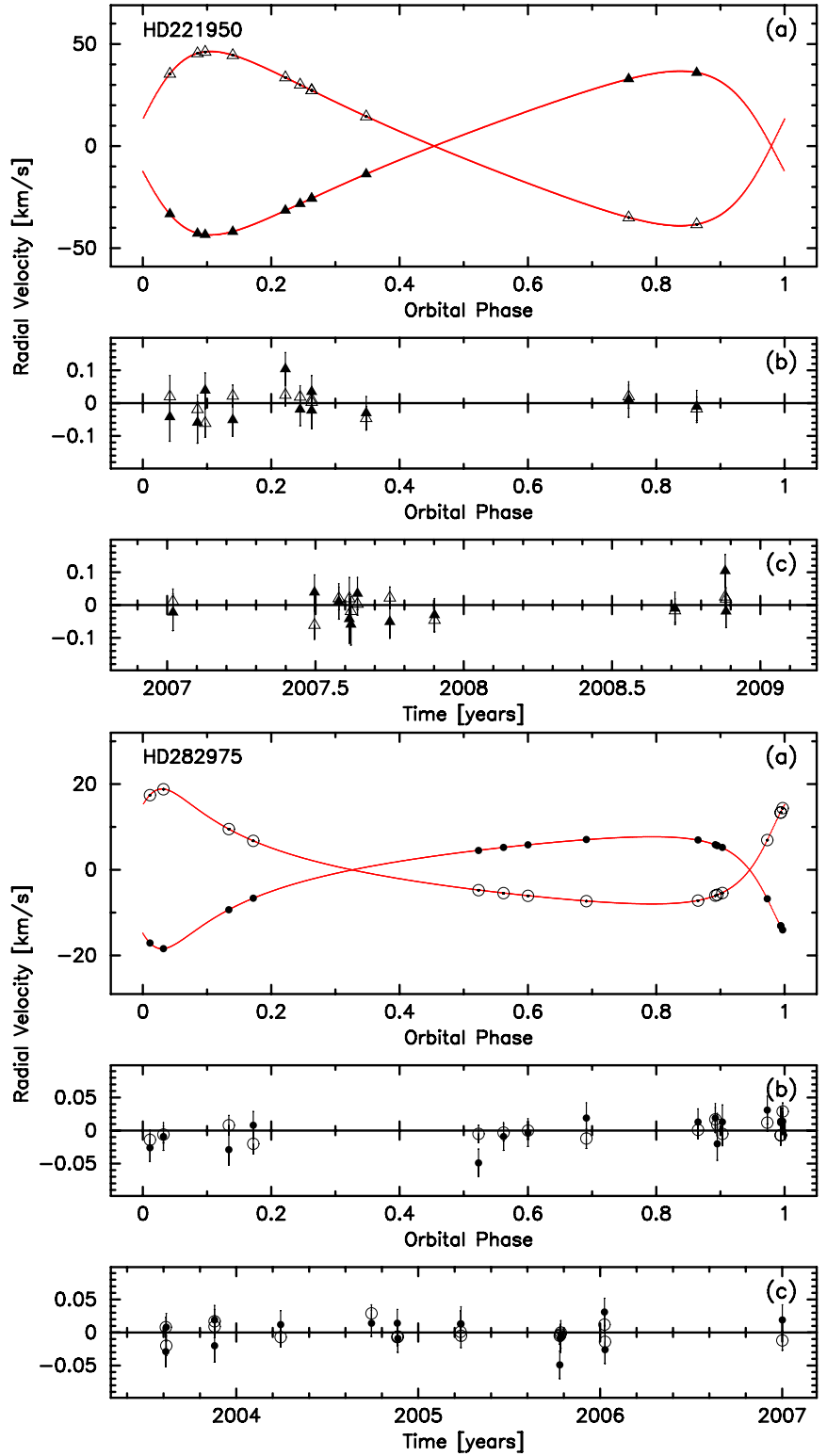

Figure 5. RVs of HD221950 (top) and HD282975 (bottom) as a function of the orbital phase (a), and the residuals (observed minus modeled RVs) as a function of the orbital phase (b) and time (c). The primary is denoted with filled symbols, the secondary with open ones, and the best-fit RV model with a solid line. The Keck I/Hires is denoted with circles, Shane/CAT/Hamspec with triangles, and TNG/Sarg with stars.

(A color version of this figure is available in the online journal.)

Keplerian RV model and a least-squares orbital fit is made simultaneously to the velocities of the primaries and secondaries (the orbital solutions and RVs will be published separately). The residuals are then inspected for planetary signatures which would obviously be the same for a primary and secondary. In the process, we have not found any clear planetary signatures.

The procedure of Cumming et al. (1999) for evaluating the regions in mass-period space in which companions can be ruled out was modified for application to the fit residuals to the twobody Keplerian orbits of the target binaries in order to search for and place limits on additional components as follows. This implementation of the analysis algorithm was thoroughly tested during the SIM Double-Blind Test (Traub et al. 2009a, 2009b).

A grid of potential companion orbital periods is sampled logarithmically at values of $P=2 f T / I$, where $f$ is an optional
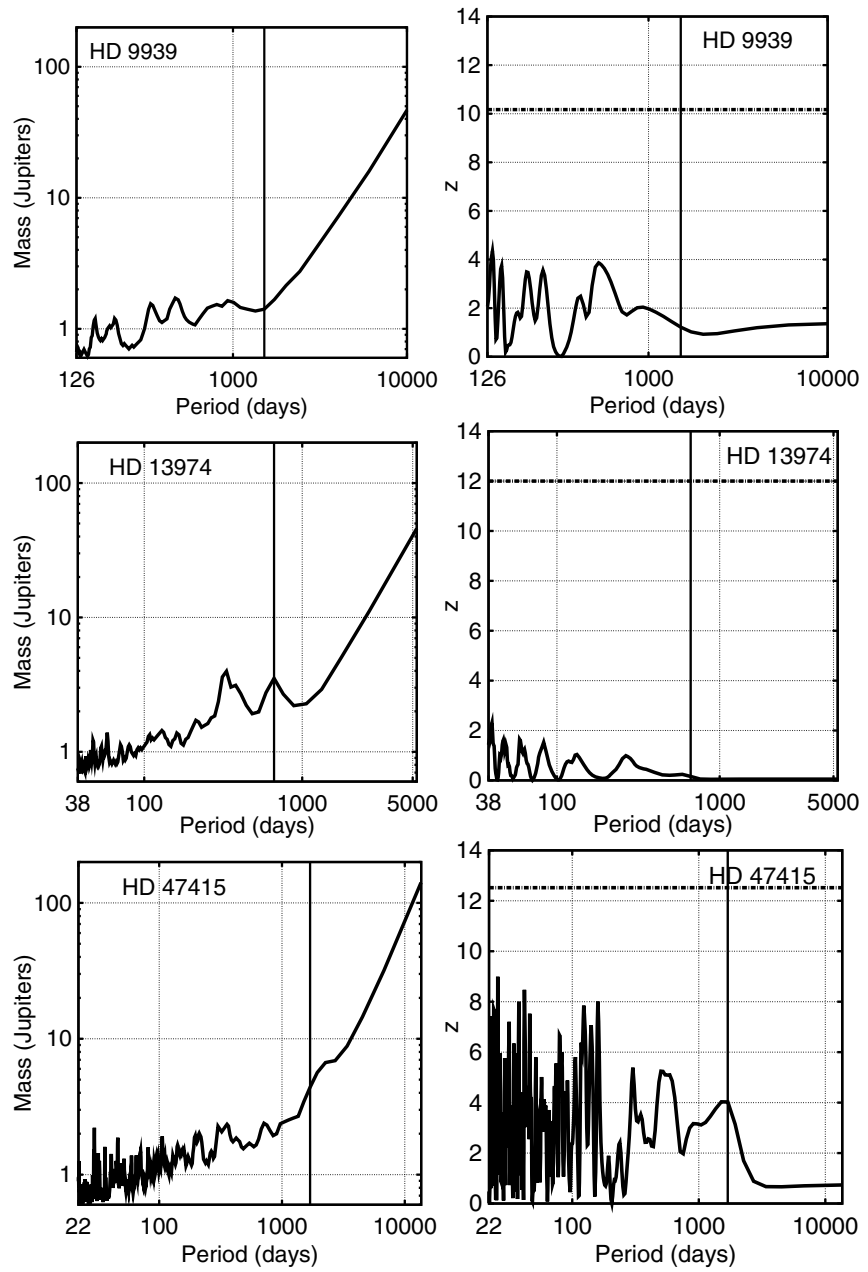

Figure 6. Planet detection limits in the $\log P-\log M$ space (orbital period-mass; left panels) and periodograms (right panels). The solid line in the left panels is a planet detection limit corresponding to the $99 \%$ confidence level. The vertical line near the orbital period of 1000 days denotes the time span of the data set.

excess factor (here 4) for finer sampling, $T$ is the time span of the observations, and $I$ is an integer beginning at 1 and continuing through that at which the sampled period is $\sim 5 x$ the binary orbital period (at which point many companion orbits are dynamically unstable). The exact limiting orbital periods shown for each star below were calculated using the Equation (3) from Holman \& Wiegert (1999). At each sampled orbital period, the RV residuals for each star are fit to a Keplerian orbit with floating offsets as

$$
v=A \cos 2 \pi t / P+B \sin 2 \pi t / P+v_{\circ, i},
$$

where $v_{\circ, i}$ is a floating velocity offset (the $i$ representing that different floating offsets are used for each star and each observatory; e.g., for binary stars with Keck data from before and after the detector upgrade, TNG-SARG data and Lick data, a total of 8 different velocity offset parameters are used). At each sample period, the $\chi^{2}$-minimizing values of $A, B$, and $v_{\circ, i}$ and resulting $\chi^{2}$ value are evaluated. The circular orbit model is found to be sensitive to mildly eccentric companions, as in Cumming et al. (1999).

The $\chi^{2}$ values of the best-fit circular orbits were recorded for each potential companion period. These were converted to the $z$-statistic of Cumming et al. (1999) for plotting signal power in 

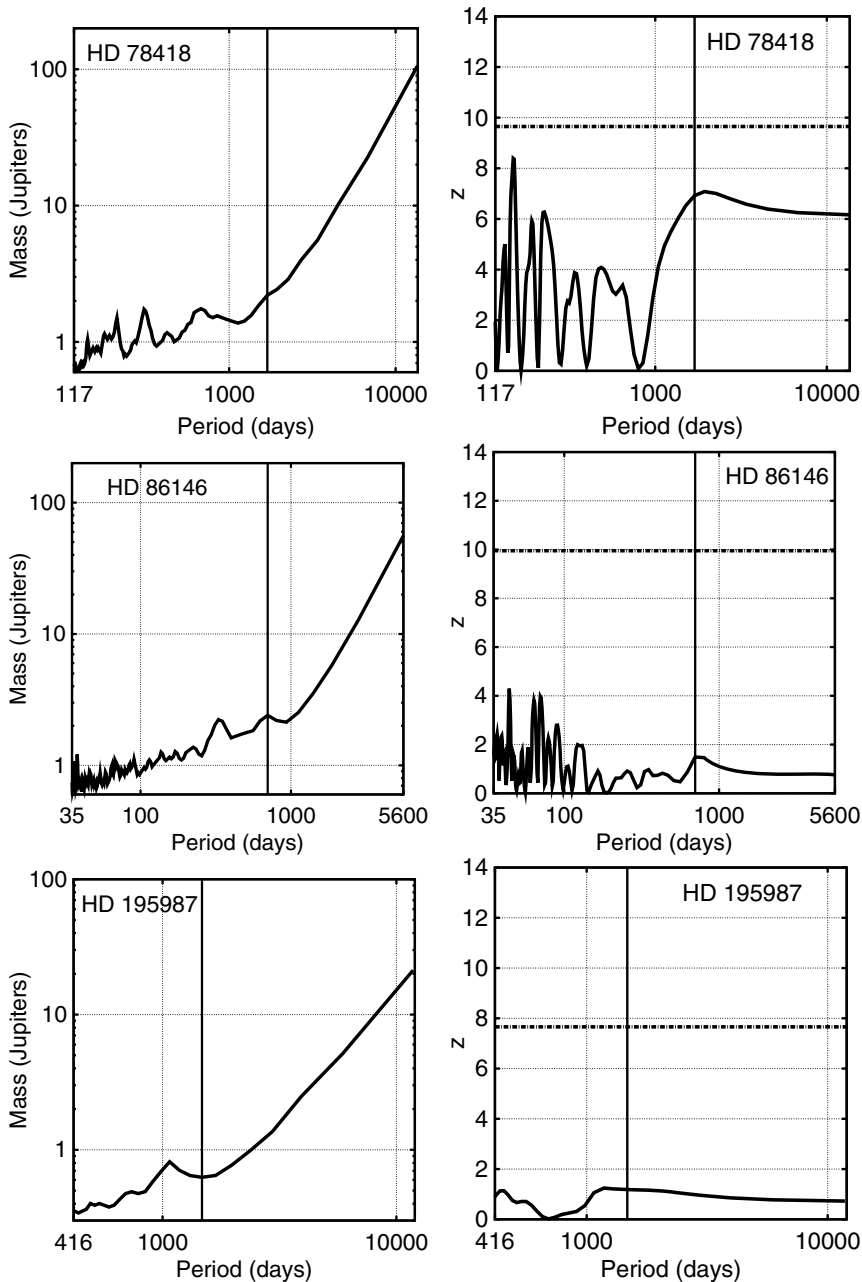

Figure 7. Planet detection limits in the $\log P-\log M$ space (orbital period-mass; left panels) and periodograms (right panels). The solid line in the left panels is a planet detection limit corresponding to the $99 \%$ confidence level. The vertical line near the orbital period of 1000 days denotes the time span of the data set.

a periodogram as

$$
\begin{gathered}
z=Q \frac{\chi_{\circ}^{2}-\chi^{2}}{\chi_{\text {min }, \text { circ }}^{2}}, \\
Q_{\text {circ }}=\frac{N_{D}-\left(2+N_{v_{\circ}, i}\right)}{2},
\end{gathered}
$$

where $N_{D}$ is twice the number of double-RV measurements, $N_{v_{\circ}, i}$ is the number of independent velocity offsets $v_{\circ, i}, \chi_{\circ}^{2}$ is the fit to no orbit at all $\left(v=v_{\circ, i}\right)$. (When making comparisons for calculating false alarm probabilities and detection limits, the $Q$ coefficients cancel and their actual values have little impact.)

Once the maximum value of $z$ has been found for the entire data set, the FAP of that largest signal is calculated as follows. A Gaussian random number generator is used to create 10,000 synthetic data sets with no Keplerian signal but with the same cadence and measurement uncertainties as the original data. This introduces a slight difference with the procedure of Cumming et al. (1999): a random number generator is used instead of scrambling and rescaling the fit residuals. As noted by those authors, the difference in these approaches causes little change in the results. These synthetic zero-signal data sets are analyzed with the same procedure as for the original data residuals.
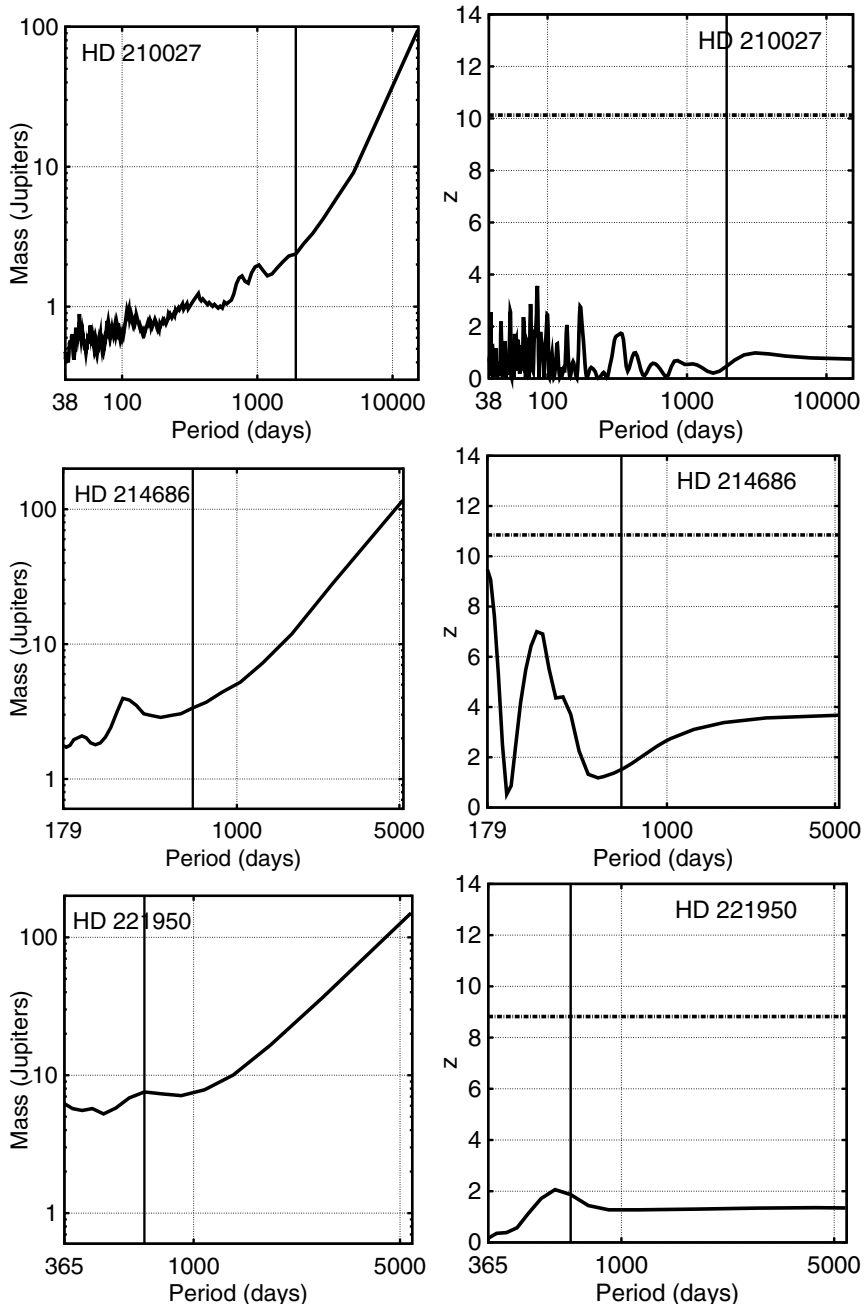

Figure 8. Planet detection limits in the $\log P-\log M$ space (orbital period-mass; left panels) and periodograms (right panels). The solid line in the left panels is a planet detection limit corresponding to the $99 \%$ confidence level. The vertical line near the orbital period of 1000 days denotes the time span of the data set.
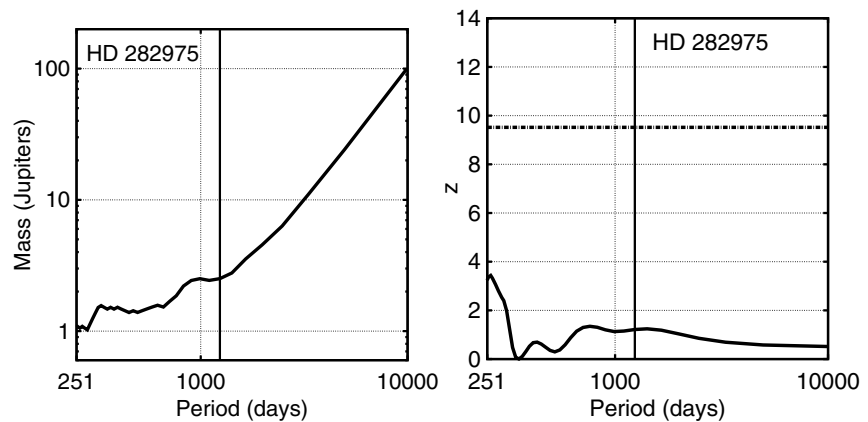

Figure 9. Planet detection limits in the $\log P-\log M$ space (orbital period-mass; left panels) and periodograms (right panels). The solid line in the left panels is a planet detection limit corresponding to the $99 \%$ confidence level. The vertical line near the orbital period of 1000 days denotes the time span of the data set.

Because these synthetic data sets are known to contain no real signal, the fraction of these whose analysis show power larger than the maximum $z$ of the real data set determines how likely that most significant value is to be a false positive.

Finally, the threshold companion RV signal (as a function of orbital period) that can be excluded using the current data residuals is evaluated. At each sample period, an initial guess for the RV amplitude $K$ that can be excluded is made, and 10,000 
Table 1

Targets and Their Radial Velocity Data Sets

\begin{tabular}{|c|c|c|c|c|c|c|c|c|c|c|}
\hline Parameter & HD 9939 & HD 13974 & HD 47415 & HD 78418 & HD 86146 & HD 195987 & HD 210027 & HD 214686 & HD 221950 & HD 282975 \\
\hline$V(\mathrm{mag})$ & 6.99 & 4.9 & 6.38 & 5.98 & 5.12 & 7.09 & 3.76 & 6.89 & 5.70 & 10.0 \\
\hline $\mathrm{Sp}$ & K0IV & G0V & F5V/F5V & G5IV-V & F5V/G0V & $\mathrm{G} 3 \mathrm{~V} / \mathrm{K} 2 \mathrm{~V}$ & F5V/G8V & F7V/F7V & F6V & G6V \\
\hline$r$ & 6.3 & 6.2 & 1.7 & 2.3 & 2.5 & 6.7 & 12 & 1 & 1.25 & 1.1 \\
\hline$M_{1}\left(M_{\odot}\right)$ & $1.072^{\mathrm{a}}$ & $0.6^{\mathrm{b}}$ & $1.4^{\mathrm{c}}$ & $1.15^{\mathrm{c}}$ & $1.35^{\mathrm{d}}$ & $0.844^{\mathrm{e}}$ & $1.326^{\mathrm{f}}$ & $1.25^{\mathrm{g}}$ & $1.31^{\mathrm{g}}$ & $1.0^{\mathrm{h}}$ \\
\hline$M_{2}\left(M_{\odot}\right)$ & $0.8383^{\mathrm{a}}$ & $0.5^{\mathrm{b}}$ & $1.2^{\mathrm{c}}$ & $1.0^{\mathrm{c}}$ & $1.08^{\mathrm{d}}$ & $0.665^{\mathrm{e}}$ & $0.819^{f}$ & $1.25^{\mathrm{g}}$ & $1.24^{\mathrm{g}}$ & $0.9^{h}$ \\
\hline$P_{\text {orbital }}$ (days) & 25.2 & 10.0 & 5.7 & 19.4 & 9.3 & 57 & 10.2 & 21.7 & 45.5 & 26 \\
\hline$a_{\text {stable }}(\mathrm{AU})$ & 0.61 & 0.23 & 0.21 & 0.61 & 0.28 & 1.25 & 0.29 & 0.85 & 1.37 & 0.97 \\
\hline$P_{\text {stable }}$ (days) & 126 & 38 & 22 & 117 & 35 & 416 & 38 & 179 & 365 & 251 \\
\hline \multicolumn{11}{|l|}{ All RVs } \\
\hline$N_{1}+N_{2}$ & 34 & 28 & 44 & 50 & 62 & 50 & 146 & 22 & 22 & 32 \\
\hline$T_{\text {span }}($ days $)$ & 1513 & 663 & 1695 & 1695 & 701 & 1474 & 1925 & 646 & 681 & 1238 \\
\hline $\mathrm{rms}_{1}\left(\mathrm{~m} \mathrm{~s}^{-1}\right)$ & 19.5 & 22.5 & 12.8 & 11.5 & 15.2 & 11.0 & 17.2 & 14.6 & 48.4 & 21.9 \\
\hline $\mathrm{rms}_{2}\left(\mathrm{~m} \mathrm{~s}^{-1}\right)$ & 36.0 & 111.1 & 25.6 & 25.0 & 67.6 & 48.0 & 85.2 & 14.7 & 29.8 & 12.5 \\
\hline \multicolumn{11}{|l|}{ Keck I/Hires } \\
\hline$N_{1}+N_{2}$ & 20 & $\ldots$ & 30 & 26 & $\ldots$ & 22 & 104 & $\ldots$ & $\ldots$ & 32 \\
\hline $\mathrm{rms}_{1}\left(\mathrm{~m} \mathrm{~s}^{-1}\right)$ & 6.8 & $\ldots$ & 12.8 & 9.9 & $\ldots$ & 2.3 & 16.5 & $\ldots$ & $\ldots$ & 21.9 \\
\hline $\mathrm{rms}_{2}\left(\mathrm{~m} \mathrm{~s}^{-1}\right)$ & 21.4 & $\ldots$ & 10.6 & 14.7 & $\ldots$ & 24.8 & 89.0 & $\ldots$ & $\ldots$ & 12.5 \\
\hline$\sigma_{1}\left(\mathrm{~m} \mathrm{~s}^{-1}\right)$ & $1-4$ & $\ldots$ & $4-11$ & $2-6$ & $\ldots$ & $3-6$ & $5-32$ & $\ldots$ & $\ldots$ & $4-17$ \\
\hline$\sigma_{2}\left(\mathrm{~m} \mathrm{~s}^{-1}\right)$ & $4-10$ & $\ldots$ & $5-11$ & $4-10$ & $\ldots$ & $7-18$ & $12-65$ & $\ldots$ & $\ldots$ & $7-15$ \\
\hline$\epsilon_{1}\left(\mathrm{~m} \mathrm{~s}^{-1}\right)$ & 6 & $\cdots$ & 11 & 5.5 & $\cdots$ & 0 & 12 & $\cdots$ & $\cdots$ & 20 \\
\hline$\epsilon_{2}\left(\mathrm{~m} \mathrm{~s}^{-1}\right)$ & 21 & $\cdots$ & 8 & 4 & $\cdots$ & 20 & 85 & $\cdots$ & $\cdots$ & 11 \\
\hline \multicolumn{11}{|c|}{ Shane/CAT/Hamspec } \\
\hline$N_{1}+N_{2}$ & 14 & 28 & 14 & 24 & 62 & 18 & 30 & 22 & 22 & $\ldots$ \\
\hline $\mathrm{rms}_{1}\left(\mathrm{~m} \mathrm{~s}^{-1}\right)$ & 14.0 & 22.5 & 12.3 & 12.0 & 15.2 & 14.2 & 20.4 & 14.6 & 48.4 & $\ldots$ \\
\hline $\mathrm{rms}_{2}\left(\mathrm{~m} \mathrm{~s}^{-1}\right)$ & 57.3 & 111.1 & 37.3 & 31.0 & 67.6 & 72.2 & 85.5 & 14.7 & 29.8 & $\ldots$ \\
\hline$\sigma_{1}\left(\mathrm{~m} \mathrm{~s}^{-1}\right)$ & $4-14$ & $7-38$ & $5-21$ & $4-27$ & $6-34$ & $7-17$ & $7-26$ & $11-26$ & $7-56$ & $\cdots$ \\
\hline$\sigma_{2}\left(\mathrm{~m} \mathrm{~s}^{-1}\right)$ & $9-73$ & $13-91$ & $8-39$ & $6-35$ & $13-63$ & $20-53$ & $16-100$ & $9-27$ & $8-55$ & $\ldots$ \\
\hline$\epsilon_{1}\left(\mathrm{~m} \mathrm{~s}^{-1}\right)$ & 20 & 19 & 8 & 11 & 7 & 13 & 18.5 & 0 & 48 & $\ldots$ \\
\hline$\epsilon_{2}\left(\mathrm{~m} \mathrm{~s}^{-1}\right)$ & 30 & 110 & 30 & 37 & 58 & 50 & 68.0 & 8 & 32 & $\ldots$ \\
\hline \multicolumn{11}{|l|}{ TNG/Sarg } \\
\hline$N_{1}+N_{2}$ & $\ldots$ & $\ldots$ & $\ldots$ & $\ldots$ & $\ldots$ & 10 & 12 & $\ldots$ & $\ldots$ & $\ldots$ \\
\hline $\mathrm{rms}_{1}\left(\mathrm{~m} \mathrm{~s}^{-1}\right)$ & $\ldots$ & $\ldots$ & $\ldots$ & $\ldots$ & $\ldots$ & 5.1 & 19.4 & $\ldots$ & $\ldots$ & $\ldots$ \\
\hline $\mathrm{rms}_{2}\left(\mathrm{~m} \mathrm{~s}^{-1}\right)$ & $\ldots$ & $\ldots$ & $\ldots$ & $\ldots$ & $\ldots$ & 15.8 & 89.9 & $\ldots$ & $\ldots$ & $\ldots$ \\
\hline$\sigma_{1}\left(\mathrm{~m} \mathrm{~s}^{-1}\right)$ & $\ldots$ & $\ldots$ & $\ldots$ & $\ldots$ & $\ldots$ & $6-12$ & $10-20$ & $\ldots$ & $\ldots$ & $\ldots$ \\
\hline$\sigma_{2}\left(\mathrm{~m} \mathrm{~s}^{-1}\right)$ & $\ldots$ & $\ldots$ & $\ldots$ & $\ldots$ & $\ldots$ & $11-17$ & $45-72$ & $\ldots$ & $\ldots$ & $\ldots$ \\
\hline$\epsilon_{1}\left(\mathrm{~m} \mathrm{~s}^{-1}\right)$ & $\ldots$ & $\ldots$ & $\ldots$ & $\ldots$ & $\ldots$ & 0 & 12 & $\ldots$ & $\ldots$ & $\ldots$ \\
\hline$\epsilon_{2}\left(\mathrm{~m} \mathrm{~s}^{-1}\right)$ & $\ldots$ & $\ldots$ & $\ldots$ & $\ldots$ & $\ldots$ & 36 & 38 & $\ldots$ & $\ldots$ & $\ldots$ \\
\hline
\end{tabular}

Notes. HD13974 is somewhat challenging to disentangle as the RV amplitudes are only 10.2 and $14.2 \mathrm{~km} \mathrm{~s}^{-1}$ for the primary and the secondary, respectively. Its mass seems to be low by a factor of 2, but we are using this published value anyways. HD221950 has relatively wide spectral lines compared to the remaining targets in this sample, and this presumably has an impact on the tomographic disentangling and the final RV precision.

References. (1) Boden et al. 2006; (2) Hummel et al. 1995; (3) de Medeiros \& Udry 1999; (4) Batten \& Morbey 1980; (5) Torres et al. 2002; (6) Boden et al. 1999;

(7) Tomkin \& Fekel 2008; (8) Mermilliod et al. 1992.
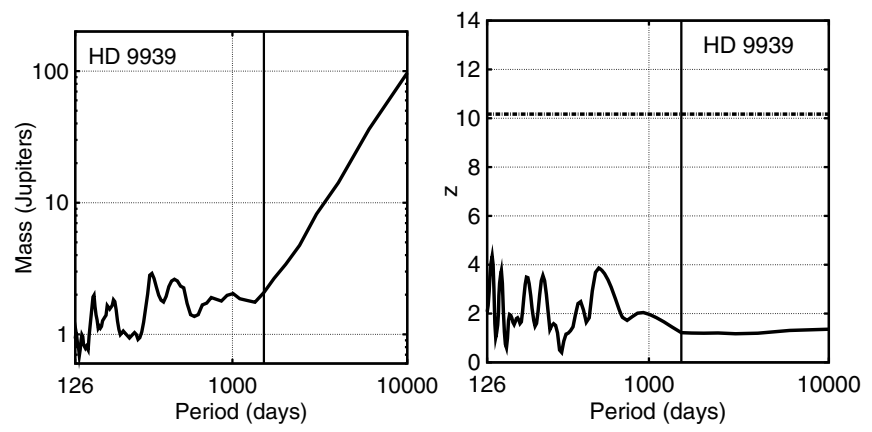

Figure 10. Planet detection limits in the $\log P-\log M$ space (orbital periodmass; left panels) and periodograms (right panels) for HD9939 and planetary orbits with the eccentricities up to 0.6 . The solid line in the left panels is a planet detection limit corresponding to the $99 \%$ confidence level. The vertical line near the orbital period of 1000 days denotes the time span of the data set.

synthetic data sets with a signal of that amplitude are generated. The orbital phase is selected randomly with flat distribution. The fraction of orbits with $z$ exceeding that of the data is computed, the RV amplitude $K$ is modified, and the procedure is iterated until the fraction is constrained near the desired reliability value (here 99\%). In Figures 6-9 (right panels), the $z$ periodograms are shown for each binary, with a horizontal line at the $1 \%$ FAP level as determined from the signal-free synthetic data sets. In Figures 6-9 (left panels), the mass $(\sin i)$-period phase space in which circumbinary companions can be excluded at the $99 \%$ confidence level are shown, where the values of mass $(\sin i)$ are calculated from the threshold companion RV amplitudes $K$ using the total masses of the binary, as estimated for each system. We have also tested orbits with non-zero eccentricities. It turns out that moderate eccentricities make little impact as the threshold lines move up by a factor of about 1.5. This is demonstrated on the case of HD9939 in Figure 10.

The targets and their RV data sets are summarized in Table 1 where Sp denotes the target's spectral type (either combined or for each component), $M_{1,2}$ the masses of the primary and secondary used in the analysis, $P_{\text {orbital }}$ the orbital period. $a_{\text {stable }}$ and $P_{\text {stable }}$ are the semimajor axis and orbital period of the first stable orbit of a circumbinary planet computed using 
the Equation (3) from Holman \& Wiegert (1999). $N_{1,2}$ denotes the number of available RV measurements for the entire data set and each subset, $\mathrm{rms}_{1,2}$ the corresponding best-fit rms from a Keplerian orbit for the entire data set as well as subsets, $T_{\text {span }}$ the time span of the entire RV data set, $\sigma_{1,2}$ are the formal RV errors, and $\epsilon_{1,2}$ are the additional errors added in quadrature.

\section{CONCLUSIONS}

Our novel iodine cell based RV technique allows one to measure precise RVs of the components of double-lined spectroscopic binary stars. With this technique, in 2003 we have initiated TATOOINE, a radial velocity search for circumbinary planets around a sample of $\sim 50 \mathrm{SB} 2 \mathrm{~s}$. In this paper, we present the first results from this survey-a non-detection of exoplanets in the $0.3-3 M_{\text {Jup }}$ regime with the orbital periods of up to 5.3 years around 10 SB2s.

Recently, two circumbinary planets around an eclipsing binary HW Vir (Lee et al. 2009) and a circumbinary brown dwarf around an eclipsing binary HS0705+6700 (Qian et al. 2009) have been claimed to be detected by means of eclipse timing. This is however not the first time when substellar companions or planets have been detected with a timing technique. In addition to the confirmed case of the three rocky planets around a millisecond pulsar B1257+12 (Konacki \& Wolszczan 2003), planets have been claimed to orbit a pulsar B0329+54 based on the timing of its radio pulses (Demianski \& Proszynski 1979; Shabanova 1995). Later, it was demonstrated that the timing variation is quasi-periodic and is not due to planets (Konacki et al. 1999). One is left to wonder if the two cases of the eclipse timing variations are indeed best explained by a periodic signal due to circumbinary bodies and not an unrecognized quasiperiodic phenomenon mimicking a periodic planetary signal.

Our sample is too small to risk any decisive conclusion about the frequency of circumbinary planets. This reminds us of the pioneering search for planets around single stars by Campbell et al. (1988). In particular, their non-detection of the population of hot Jupiters. We also have not detected any short period planets or to be precise planets with periods near the inner orbital stability limits. The question of the existence of such circumbinary planets and hence the impact of the central body's binarity on the parking mechanisms for migrating planets remains open. It should also be noted that even though all our targets are SB2s, they constitute quite a diverse sample. Their orbital periods range from 5.7 to 57 days and the primary to secondary mass ratios range from 1 to 1.6. This presumably should make an impact on the formation and evolution of circumbinary planets. The targets from RV surveys for planets around single stars are more homogenous and provide for an overall similar environment.

When comparing our planet detection limits to those from the Anglo-Australian Planet Search (O'Toole et al. 2009), the McDonald Observatory Planet Search (Wittenmyer et al. 2006), and the Keck Planet Search (Cumming et al. 2008; all employing iodine cells), one will notice that these surveys allow for a detection of up to several times less massive planets. This is due to a higher precision (now approaching $1 \mathrm{~m} \mathrm{~s}^{-1}$ in many cases), typically larger number of RV measurements and a longer time span of the data sets. On the other hand, the comparison with the planet detection limits for the Lick Planet Search (Cumming et al. 1999), the first RV survey to employ an iodine cell, demonstrates that our survey performs similarly. One should also remember that the mass of the central body in our survey is typically $1.5-2.0$ times higher then in the case of single stars which obviously decreases the sensitivity to planets in terms of masses by such a factor.

Our search continues and the changes to the method are constantly being made to improve the RV precision. Theoretical works on the formation and evolution of circumbinary planets are strongly encouraged as now we have the observational tools to test them.

We thank Lucasfilm Ltd for inspiring the TATOOINE planet search (and careers of many of us), and Lucasfilm's Senior Director of Business Affairs David Anderman for an excellent tour of the Lucasfilm complex upon hearing about our program. The tour of Lucasfilm was a highlight of the undergraduate research experiences of Agnieszka Czeszumska, Sam Halverson, Tony Mercer, and Jackie Schwehr. We thank the California and Carnegie Exoplanet Search team, and Geoff Marcy in particular, for allowing us to access their precision velocimetry tools at Lick Observatory. This research has made use of the Simbad database, operated at CDS, Strasbourg, France. M.W.M. acknowledges support from the Townes Fellowship Program. M.K. is supported by the Foundation for Polish Science through a FOCUS grant and fellowship, by the Polish Ministry of Science and Higher Education through grants N203 005 32/0449 and 1P03D-021-29. Part of the algorithms used in this analysis were developed during the SIM Double-Blind Test, under JPL contract 1336910. This research has made use of the Simbad database, operated at CDS, Strasbourg, France. The observations on the TNG/SARG have been funded by the Optical Infrared Coordination Network (OPTICON), a major international collaboration supported by the Research Infrastructures Programme of the European Commissions Sixth Framework Programme.

Facilities: Keck I/Hires, TNG/Sarg, Shane/Hamspec

\section{REFERENCES}

Andersen, J., Lindgren, H., Hazen, M. L., \& Mayor, M. 1989, A\&A, 219, 142 Artymowicz, P., \& Lubow, S. H. 1994, ApJ, 421, 651

Batten, A. H., \& Morbey, C. L. 1980, PASP, 92, 98

Boden, A. F., Torres, G., \& Latham, D. W. 2006, ApJ, 644, 1193

Boden, A. F., et al. 1999a, ApJ, 515, 356

Campbell, B., Walker, G. A. H., \& Yang, S. 1988, ApJ, 331, 902

Colavita, M. M., et al. 1999, ApJ, 510, 505

Cumming, A., Butler, R. P., Marcy, G. W., Vogt, S. S., Wright, J. T., \& Fischer, D. A. 2008, PASP, 120, 531

Cumming, A., Marcy, G. W., \& Butler, R. P. 1999, ApJ, 526, 890

de Medeiros, J. R., \& Udry, S. 1999, A\&A, 346, 532

Demianski, M., \& Proszynski, M. 1979, Nature, 282, 383

Diego, F., Charalambous, A., Fish, A. C., \& Walker, D. D. 1990, Proc. SPIE, 1235,562

Dutrey, A., Guilloteau, S., \& Simon, M. 1994, A\&A, 286, 149

Dvorak, R. 1984, Celest. Mech, 34, 369

Gratton, R. G., et al. 2001, Exp. Astron., 12, 107

Holman, M. J., \& Wiegert, P. A. 1999, AJ, 117, 621

Hummel, C. A., Armstrong, J. T., Buscher, D. F., Mozurkewich, D., Quirrenbach, A., \& Vivekanand, M. 1995, AJ, 110, 376

Kastner, J. H., Zuckerman, B., Hily-Blant, P., \& Forveille, T. 2008, A\&A, 492, 469

Konacki, M. 2005a, ApJ, 626, 431

Konacki, M. 2005b, Nature, 436, 230

Konacki, M. 2009, IAU Symp., 253, 141

Konacki, M., \& Lane, B. F. 2004, ApJ, 610, 443

Konacki, M., Lewandowski, W., Wolszczan, A., Doroshenko, O., \& Kramer, M. 1999, ApJ, 519, L81

Konacki, M., \& Wolszczan, A. 2003, ApJ, 591, L147

Kurucz, R. L. 1995, in ASP Conf. Ser. 78, Astrophysical Applications of Powerful New Databases, ed. S. J. Adelman \& W. L. Wiese (San Francisco, CA: ASP), 205 
Lee, J. W., Kim, S.-L., Kim, C.-H., Koch, R. H., Lee, C.-U., Kim, H.-I., \& Park, J.-H. 2009, AJ, 137, 3181

Marcy, G. W., \& Butler, R. P. 1992, PASP, 104, 270

Mathieu, R. D., Adams, F. C., \& Latham, D. W. 1991, AJ, 101, 2184

Mathieu, R. D., Stassun, K., Basri, G., Jensen, E. L. N., Johns-Krull, C. M., Valenti, J. A., \& Hartmann, L. W. 1997, AJ, 113, 1841

Mermilliod, J.-C., Rosvick, J. M., Duquennoy, A., \& Mayor, M. 1992, A\&A, 265,513

Moriwaki, K., \& Nakagawa, Y. 2004, ApJ, 609, 1065

Muterspaugh, M. W. 2005, PhD thesis, MIT

Muterspaugh, M. W., Konacki, M., Lane, B. F., \& Pfahl, E. 2007, arXiv:0705.3072

Muterspaugh, M. W., Lane, B. F., Kulkarni, S. R., Burke, B. F., Colavita, M. M., \& Shao, M. 2006, ApJ, 653, 1469

Ofir, A. 2008, MNRAS, 387, 1597

Ofir, A. 2009, in IAU Symp. 253, Transiting Planets, ed. F. Pont, D. Queloz, \& D. Sasselov (Cambridge: Cambridge Univ. Press), 378

O’Toole, S. J., Tinney, C. G., Jones, H. R. A., Butler, R. P., Marcy, G. W., Carter, B., \& Bailey, J. 2009, MNRAS, 392, 641

Pierens, A., \& Nelson, R. P. 2007, A\&A, 472, 993
Pierens, A., \& Nelson, R. P. 2008a, A\&A, 478, 939

Pierens, A., \& Nelson, R. P. 2008b, A\&A, 483, 633

Qian, S.-B., et al. 2009, ApJ, 695, L163

Quintana, E. V., \& Lissauer, J. J. 2006, Icarus, 185, 1

Schneider, J. 1994, Planet. Space Sci., 42, 539

Schneider, J., \& Chevreton, M. 1990, A\&A, 232, 251

Scholl, H., Marzari, F., \& Thébault, P. 2007, MNRAS, 380, 1119

Shabanova, T. V. 1995, ApJ, 453, 779

Tomkin, J., \& Fekel, F. C. 2008, AJ, 135, 555

Torres, G., Boden, A. F., Latham, D. W., Pan, M., \& Stefanik, R. P. 2002, AJ, 124, 1716

Traub, W. A., et al. 2009a, Bull. Am. Astron. Soc., 41, 267

Traub, W. A., et al. 2009b, arXiv:0904.0822

Vogt, S. S. 1987, PASP, 99, 1214

Vogt, S. S., et al. 1994, Proc. SPIE, 2198, 362

Wittenmyer, R. A., Endl, M., Cochran, W. D., Hatzes, A. P., Walker, G. A. H., Yang, S. L. S., \& Paulson, D. B. 2006, AJ, 132, 177

Zucker, S., \& Mazeh, T. 1994, ApJ, 420, 806

Zuckerman, B., Fekel, F. C., Williamson, M. H., Henry, G. W., \& Muno, M. P. 2008, ApJ, 688, 1345 